\title{
A Machine-learning Signature Performs as Well as Experts' Consensus to Diagnose Pelvic Lymph Node Involvement in Bladder Cancer on [ $\left.{ }^{18} \mathrm{~F}\right]-\mathrm{FDG}$ PET/CT: A Pathology-controlled Study
}

Antoine GIRARD ( $\nabla$ a.girard@rennes.unicancer.fr )

Centre Eugene Marquis https://orcid.org/0000-0002-9472-9980

\section{Laurent DERCLE}

Columbia University Medical Center: Columbia University Irving Medical Center

Helena VILA-REYES

Columbia University Medical Center: Columbia University Irving Medical Center

\section{Lawrence H. SCHWARTZ}

Columbia University Medical Center: Columbia University Irving Medical Center

\section{Astrid GIRMA}

Hospital Foch - Suresnes: Hopital Foch

Marc BERTAUX

Hospital Foch - Suresnes: Hopital Foch

Camelia RADULESCU

Hospital Foch - Suresnes: Hopital Foch

Thierry LEBRET

Hospital Foch - Suresnes: Hopital Foch

Olivier DELCROIX

University Hospital Centre Brest: CHRU de Brest

Mathieu ROUANNE

Hospital Foch - Suresnes: Hopital Foch

\section{Keywords:}

Posted Date: May 18th, 2021

DOI: https://doi.org/10.21203/rs.3.rs-520536/v1

License: (c) (1) This work is licensed under a Creative Commons Attribution 4.0 International License. Read Full License 


\section{Abstract}

The authors have requested that this preprint be removed from Research Square. 